\title{
Metabolism and Metabolomics by MRS
}

\author{
Yuen-Li Chung $^{1}$, Madhu Basetti ${ }^{2}$, John R Griffiths ${ }^{2}$ \\ ${ }^{1}$ Cancer Research UK Cancer Imaging Centre, Division of Radiotherapy \& Imaging, The \\ Institute of Cancer Research, 123 Old Brompton Road, London SW7 3RP, UK \\ ${ }^{2}$ Cancer Research UK Cambridge Research Institute, University of Cambridge, Li Ka Shing \\ Centre, Robinson Way, Cambridge CB2 0RE, UK
}

\begin{abstract}
The main use of MRS is for the assessment of metabolism in vivo; that is because it has the unique ability to monitor metabolism noninvasively without the use of ionizing radiation. In recent years MRS has also become widely used for metabolomics, the science that aspires to monitor the metabolome - the totality of small-molecule metabolites in an organism, a cell or a disease. This chapter discusses the properties of MRS that make it suitable for metabolomic studies and the reasons why they are mainly performed ex vivo, as well as the methods used for preparing samples, performing the MRS and analyzing the resulting data. Further sections discuss MRS detection of metabolic aspects of the common cellular processes apoptosis, necrosis and autophagy. A final section summarizes metabolic studies of some diseases by MRS: cancer, cardiovascular disease, neurological and neurodegenerative diseases, and muscle disease.
\end{abstract}

Keywords: Metabolism; Metabolomics; Metabolome; HR-MAS MRS; Tissue Extraction; In Vivo MRS; Ex Vivo MRS 


\section{INTRODUCTION}

\subsection{Metabolism and Metabolomics}

Most of this book is concerned with the use of MRS to study aspects of metabolism in vivo, a situation where the MRS method has the unique advantage of detecting and quantifying metabolites non-invasively without the use of ionizing radiation. Over the last 15 years however, a related topic has developed - "Metabolomics" - and this too has been studied by MRS methods. Whereas metabolic biochemistry usually focusses on individual reactions or pathways, metabolomics is the study of the totality of small molecule metabolites (the "metabolome") in an organism. It has recently taken a place alongside the more familiar "omics" sciences of genomics, transcriptomics and proteomics. There are thought to be several thousand metabolites (i.e. low molecular weight chemical compounds) in a typical cell, and their concentrations at any moment in time constitute the metabolome. All of the enzymes and some of the other proteins coded by the genome ultimately have their effect on the phenotype of the organism by influencing the concentrations of its metabolites, so the metabolome is an important target of research.

Note that like the proteome and transcriptome, but unlike the genome, the metabolome is state-specific, so that one can speak for instance of the metabolome of an individual cell within an organism or the metabolome of a disease state. Furthermore, the metabolome is much less stable than even the proteome: the concentrations of metabolites within it can vary over minutes or even seconds in response to external stimuli (e.g. the stimulus to contract a muscle simultaneously initiates breakdown of glycogen as a fuel) or to homeostatic interactions between the metabolites in metabolic pathways themselves. At present it is not possible to measure 
more than a few hundred of the metabolites in the metabolome; those subsets that can be measured are often termed "metabolic profiles".

\subsection{Advantages of MRS for metabolomics}

There has been an explosion of interest in the use of NMR-based methods to study the metabolome over the last decade (see Fig 1). This is because NMR and MRS have many advantages for metabolic studies, both in vivo and ex vivo. As has already been mentioned, in vivo studies by MRS can detect metabolites non-invasively, which is often a crucial advantage for studying individual metabolic reactions. However, only a small number of metabolites present at high concentration can be measured in vivo because of the relative insensitivity of MRS at the magnetic fields that can be used and the broad spectral lines that are observed. In order to detect as many metabolites as possible, metabolomic studies are often performed by ex vivo MRS methods on samples such as tissue biopsies and cultured cells. Such spectra give higher sensitivity because higher fields and longer scan times can be employed, as well as better spectral resolution. Note that these ex vivo MRS methods are often termed "NMR" methods, since they are usually performed on vertical-bore high-resolution NMR instruments; however we will retain the term MRS since it is used elsewhere in this book, and since the two methods are fundamentally identical. We will not deal with measurements of metabolites in body fluids (see emrstm1048).

Even when used on ex vivo specimens, MRS is much less sensitive than mass spectrometry (MS), the main competing analytical method in metabolomics. However, it has some compensating advantages. Preparation of a tissue sample for MRS involves a simple extraction or, for HR-MAS (high-resolution magic angle spinning; see 
emrstm1231) MRS, simply placing the solid sample in a rotor insert. The subsequent MRS procedure then quantifies all of the metabolites in that extract or intact sample so that any quantification errors will usually cancel out and the relative amounts of the metabolites will be detected with good precision. Furthermore, the HR-MAS method is non-destructive, and even after extraction the metabolites in the sample are available for subsequent studies. MS methods, in contrast, require preliminary separation of the metabolites within the samples, usually by liquid chromatography (LC-MS) or gas chromatography (GC-MS). Before performing GC-MS it is usually necessary to derivatize the metabolites in order to make them volatile. Quantification errors may be introduced by the separation process and particularly by the derivatisation. Lastly, the MS procedure itself involves ionization of the metabolites (or their derivatives) and further errors can be introduced if ionization is incomplete.

$<$ Fig 1 near here >

Thus although ex vivo MRS methods are usually able to quantify a metabolic profile of only 20-30 high-concentration metabolites, they do so with great precision and reproducibility, an advantage that makes them particularly suited to studies in which the metabolome of a cell or organism is perturbed and then compared with the original metabolome.

\section{Ex Vivo MRS Methods for studying the metabolome}

The broad MR spectral lines observed in vivo (see Fig 2A) are also seen when spectra are obtained ex vivo from solid tissue biopsies or cultured cells. There are two solutions to that problem: HR-MAS MRS (Fig 2B) or extraction of the metabolites into solution (Fig 
2C). HR-MAS MRS involves spinning the solid sample in a rotor at a "magic angle" relative to the main magnetic field (see section 2.2 below and emrstm1231). That eliminates the broadening processes caused by dipole-dipole interactions, giving a spectrum with peaks that are adequately resolved. ${ }^{1} \mathrm{H}$ HR-MAS MRS is by far the most commonly used method here. Performing MRS on extracted metabolites in solution in a high-resolution instrument narrows the spectral linewidths still further due to the motional narrowing, as shown in Fig 2C.

\section{$<$ Fig 2 near here $>$}

\subsection{Preparation of tissue or cell extracts for MRS}

Living tissues or cultured cells can be prepared for MRS by extraction with a liquid that denatures the tissue proteins. This inactivates the enzymes that would otherwise scramble the metabolite concentrations, and also releases into solution metabolites that were bound to proteins or sequestered in organelles. The denatured proteins are spun down and the supernatant is removed, neutralized, freeze-dried and re-dissolved in $\mathrm{D}_{2} \mathrm{O}$ (deuterated water) for analysis by MRS. An external standard is normally added to the extract to permit chemical shift standardization and quantification.

Because post-mortem scrambling of metabolites and even simple chemical breakdown can occur within a few seconds of tissue death, it is routine in metabolic biochemistry to freeze tissue samples instantly by crushing them between liquidnitrogen-cooled blocks mounted on tongs ("freeze-clamping"). In cell culture work an additional problem occurs: procedures such as stripping cells from a culture plate or bottle (e.g. with trypsin) and then spinning them down in a centrifuge tube, are quite time-consuming and likely to alter the metabolism of the cells. It is best, therefore to 
remove the culture medium quickly, add the extraction medium to the culture plate or bottle and then scrape the cells directly into the denaturing liquid.

The classic extraction medium in metabolic biochemistry is $5 \%$ perchloric acid, which has the advantage that it can subsequently be removed by precipitation as potassium perchlorate. It is very efficient at denaturing enzymes but it releases artefactual chemicals from the plastic tissue culture plates or bottles ${ }^{1}$. If allowance is made for these artefacts it is still a very reliable method.

The other main class of extraction media is organic solvents. Chloroformmethanol-water 1:1:1 mixtures have been used for more than half a century to extract lipids from tissues ${ }^{2}$. A homogenate tissue and the extraction medium is centrifuged and the supernatant separated into two phases with the lipids in the lower chloroform layer, and the polar compounds (including most of the metabolites) in the upper methanol/water phase. More recently, Leibfritz's group ${ }^{3}$ developed this method into a combined extraction technique for both water-soluble metabolites and lipids. Another organic solvent that is sometimes used for tissue extraction is acetonitrile.

\subsection{HR-MAS MRS}

To obtain a metabolite spectrum from a solid tissue sample by HR-MAS MRS, it is simply necessary to pack the sample into a rotor insert. The spectrum is obtained while the sample is spun at several thousand rpm at the "magic angle" (ca. 54.74"). An advantage of this method is that no extraction procedure is required and the sample remains largely unharmed at the end of the experiment so that it can be used again, e.g. for gene expression or histological studies. 


\subsection{MRS studies of metabolite uptake and output}

MRS methods are particularly useful for measuring the uptake and output of metabolites from cultured cells. Since cell culture medium has to be changed every few days one simply freeze-dries samples of the used and unused culture media, redissolves them in $\mathrm{D}_{2} \mathrm{O}$ and obtains MRS spectra. Most cultured cells take up energy substrates such as glucose and glutamine and secrete products such as lactate. However, many other metabolites enter or leave the cells in relatively small amounts. By calculating the carbon content of the metabolites entering and leaving the cell one can perform a carbon balance study that gives an indication of the fuels used by the cells. More elaborate metabolic flux studies can be done using labelled substrates both ex vivo and in vivo (see emrstm1468).

\subsection{Metabolites that can be quantified by MRS ex vivo}

Table 1 shows the metabolites that are routinely quantifiable in ${ }^{1} \mathrm{H},{ }^{13} \mathrm{C}$ or ${ }^{31} \mathrm{P}$ MRS spectra of living tissues. This list is not exhaustive: some metabolites are found only in certain tissues: $\mathrm{N}$-acetylaspartate, for instance, is mainly found in the brain and citrate in the prostate. Intracellular metabolites in cell cultures can be quantified relative to cell numbers, cell volume or protein content; metabolites measured in tissue samples can be normalized to tissue wet weight or protein concentration.

\subsection{Pulse sequences for ex vivo MRS}

${ }^{1} \mathrm{H}$ MRS metabolite profiles are usually obtained with solvent suppression pulse sequences such as pre-saturation (e.g. pulse sequence "zgpr" on Bruker NMR software) or 1D-NOESY (nuclear Overhauser effect spectroscopy, emrstm0347). 
Other 2D-MRS methods such as COSY (correlation spectroscopy, emrstm0096), HSQC (heteronuclear single quantum coherence spectroscopy, emrstm0563) and TOCSY (total correlated spectroscopy, emrstm1165) can then be used for confirmation of the assignments of the metabolites ${ }^{4}$. The broad signals of lipids and macromolecules that appear in ${ }^{1} \mathrm{H}$ HR-MAS MRS spectra of tissue samples can be filtered out with a pre-saturated CPMG (Carr-Purcell-Meiboom-Gill) pulse sequence, which is commonly used as a $\mathrm{T}_{2}$-filter. Similarly, a diffusion-weighted filter (stimulated-echo pulse sequence with pulsed gradients - STE-PG) can be used to filter out the low-molecular weight metabolite signals from the lipid and macromolecular signals ${ }^{4}$.

\subsection{Data processing for ex vivo MRS}

Phase correction and baseline correction are routinely employed in the pre-processing of metabolite profiles. Metabolites are then initially identified by measuring their chemical shifts relative to internal standards such as trimethylsilyl propanoic acid (TSP) or 4,4-dimethyl-4-silapentane-1-sulfonic acid (DSS) Three broad methods of spectral analysis are employed in metabolomic studies.

\subsubsection{Metabolite fingerprinting (the chemometric approach)}

Most metabolomic studies on clinical specimens use chemometric methods as the matrix of data can be huge, with many dependent and independent variables, thus requiring reduction in data dimensionality. The initial pre-processing of the spectra involves normalization of the peaks to a standard, followed by "binning" (or "bucketing") of the spectrum - i.e. dividing the chemical shift range into a number of bins and measuring the area of the peaks in each bin. Binning eliminates small alterations in the chemical shifts of the peaks due to factors 
such as $\mathrm{pH}$, ionic strength or temperature. The bins should ideally be wide enough to encompass the whole of a peak but not so large that they take in more than one peak.

The spectra are then initially analyzed by unsupervised pattern recognition methods such as Principal Component Analysis (PCA, see emrstm1472) to identify general trends and outlier samples, followed by supervised methods such as Partial Least Squares-Discriminant Analysis (PLSDA) or Orthogonal Partial Least Squares-Discriminant Analysis (OPLS-DA) ${ }^{5}$ which can give better separation (see Fig 3). These methods are increasingly applied in the metabolomics field where the biomarkers (classifiers) can be useful in classifying groups (e.g. health vs disease or control vs treated) based on their metabolite profiles. Samples must be collected and processed in an identical manner for these studies.

$<$ Fig 3 near here>

\subsubsection{Quantitative metabolomics (Metabolite Profiling)}

In contrast to the chemometric approach described above, in quantitative metabolomics the metabolites are identified from metabolite libraries and quantified. Metabolites can be quantified in terms of absolute concentrations (e.g. $\mathrm{mmol} / \mathrm{l}$ or $\mathrm{mg} / \mathrm{g}$ protein) by integrating the areas under the metabolite peaks and then comparing them with that of an added internal reference compound of known concentration, making allowance for the number of spins contributing to each peak. Absolute concentrations of metabolites are useful in systems biology studies, particularly when metabolomic data need to be combined or correlated with other global profiling data (e.g. transcriptomic or proteomic data) from the 
same biological samples. This is also a better strategy than chemometrics when the samples cannot be collected and processed in an identical manner.

\subsubsection{Metabolite target analysis (Isotopomer analysis)}

This approach focuses on metabolites in a particular metabolic pathway, enabling dynamic metabolic flux analysis. Substrates labelled with ${ }^{13} \mathrm{C}$ can be traced through a metabolic pathway by ${ }^{13} \mathrm{C}$ MRS. Due to the lower sensitivity of ${ }^{13} \mathrm{C}$ MRS methods and the high costs of labelled substrates, this approach has not been very popular until recently. However, its future looks promising because of recent developments in hyperpolarized ${ }^{13} \mathrm{C}$ MRS protocols (see emrstm1457, $1468,1253)$.

\section{MRS OF CELLULAR PROCESSES}

\subsection{Apoptosis}

An accumulation of the $-\mathrm{CH}_{2}-\mathbf{C H}_{2}-\mathrm{CH}_{2}-$ lipid groups is associated with apoptosis in cancer cells in culture, but the presence of this lipid resonance has been found to coincide with areas of necrosis (rather than apoptosis) in solid tumors. The ${ }^{1} \mathrm{H}$ MRS visible lipids were found to accumulate and to increase their degree of lipid saturation in solid tumors during apoptosis. These lipids are attributed to cell constituent breakdown products that form lipid vesicles in dying cells ${ }^{6}$. Changes in phosphocholine (PC), glycerophosphocholine (GPC) and taurine, measured by ${ }^{1} \mathrm{H}$ MRS, have also been reported to be associated with apoptosis (emrstm1093).

Decreased hyperpolarized $\left[1-{ }^{13} \mathrm{C}\right]$ pyruvate to $\left[1-{ }^{13} \mathrm{C}\right]$ lactate exchange, measured by ${ }^{13} \mathrm{C}$ MRS, was found to be associated with apoptosis induced by etoposide treatment in EL-4 murine lymphoma xenografts. These changes were caused by the 
activation of poly(ADP)-ribose polymerase (PARP) during apoptosis, which led to depletion of the nicotinamide adenine dinucleotide (NAD+, NADH) $\operatorname{pool}^{7}$ (emrstm1093).

\subsection{Necrosis}

Accumulation of MR-visible lipids has been found in necrotic regions of tumors by ${ }^{1} \mathrm{H} \mathrm{MRS}^{6}$. The conversion of hyperpolarized $\left[1,4-{ }^{13} \mathrm{C}\right]$ fumarate to $\left[1,4-{ }^{13} \mathrm{C}\right]$ malate measured by ${ }^{13} \mathrm{C}$ MRS has been found to be a marker of necrosis. (emrstm1093).

\subsection{Autophagy}

Decreases in lactate secretion and in the hyperpolarized ${ }^{13} \mathrm{C}$-labelled pyruvate to lactate exchange rate are found in cells and tumors undergoing treatment- and starvation-induced autophagy. The underlying mechanisms are thought to involve an increase in the NAD+/NADH ratio in drug-induced autophagy ${ }^{8}$ and a decrease in lactate dehydrogenase activity in starvation-induced autophagy 9 .

\section{METABOLOMIC STUDIES OF DISEASES}

\subsection{Cancer Metabolomics:}

Reprogramming of energy metabolism has been defined as an emerging hallmark of cancer $^{10}$. It is distinguished by the "Warburg effect", a shift in energy production from oxidative phosphorylation to glycolysis even when oxygen is freely available. To support tumor growth and proliferation, cancer cells have increased glucose uptake, lactate production, phospholipid turnover and glutamine metabolism. Many important genetic mutations (e.g., p53, 5'adenosine monophosphate-activated protein kinase (AMPK), hypoxia-inducible factor (HIF)), oncogenes (e.g., Ras and c-Myc) and signaling pathways (e.g., PI3K/AKT/mTOR and MAPK/ERK) have been found 
to regulate these processes and to aid development and progression of cancer ${ }^{11}$. Numerous anticancer therapies, such as inhibitors of phosphoinositide 3-kinase (PI3K), protein kinase B (AKT), HIF-1, pyruvate dehydrogenase kinase, choline kinase, glucose transporter-1 (Glut-1) or monocarboxylate transporter-1 (MCT-1), target these metabolic abnormalities in cancer.

MRS methods, both in vivo and ex vivo, can be used to examine the reprogrammed tumor metabolism and signaling pathways that support tumor growth, and to aid the development of pharmacodynamic biomarkers that could potentially assess response to therapy or the emergence of tumors resistant to therapy. Such information could be useful in clinical trials and for individualising therapy ${ }^{12}$. Metabolomic MRS has also been investigated for diagnosing and grading many cancer types, for example, prostate $^{13}$ (see emrstm1433), brain $^{14}$ (emrstm1425), cervix ${ }^{15}$, lung ${ }^{16}$, and breast $^{17}$ (emrstm1424), as well as examining the effect of cancer on host organs ${ }^{18}$.

\subsubsection{Glycolytic metabolism}

Aerobic glycolysis (the Warburg effect) is a well-known phenomenon in cancer, and the rate of glycolysis appears to be associated with the differentiation status and growth rate of the tumor ${ }^{19}$. In vivo ${ }^{1} \mathrm{H}$ and ${ }^{13} \mathrm{C}$ MRS (together with ${ }^{13} \mathrm{C}$-enriched substrates) can be used non-invasively to examine and monitor the glycolytic behavior of different tumor types and their response to treatment. Lactate production can be measured in vivo by ${ }^{1} \mathrm{H}$ MRS using a multiple-quantum-coherence-edited sequence or by ${ }^{13} \mathrm{C}$ MRS using a ${ }^{13} \mathrm{C}$-enriched substrate, such as, glucose [emrstm1253], both in the steady-state and in real time (by Dynamic Nuclear 
Polarization (DNP). Lactate can be measured ex vivo by ${ }^{1} \mathrm{H}$ HR-MAS MRS on tumor biopsies or high-resolution ${ }^{1} \mathrm{H}$ MRS on extracts. In vivo ${ }^{13} \mathrm{C}$ MRS measurement of hyperpolarized ${ }^{13} \mathrm{C}$-labelled [1-pyruvate]-to-lactate exchange has been used to measure real-time flux of pyruvate to lactate non-invasively, both in preclinical models and in patients [emrstm1253].

Over-expression of the hypoxia inducible transcription factors HIF-1 and HIF-2 has been found in many cancers and can cause an increased rate of glycolysis and glucose metabolism. In vivo and ex-vivo ${ }^{1} \mathrm{H}$ MRS have been used to study the effects of these transcription factors. For example, overexpression of HIF-2 $\alpha$ resulted in tumors with higher glucose and lower lactate and alanine levels, together with faster tumor growth and increased pyruvate dehydrogenase and lower pyruvate dehydrogenase kinase-1 expressions, all indicating that these tumors maintain their growth rate by reducing glycolysis and increasing mitochondrial metabolism ${ }^{20}$.

Real-time lactate measurement by DNP ${ }^{13} \mathrm{C}$ MRS has been used to examine the effect of signaling pathway inhibition on the Warburg effect in tumors; for example, reduced $\left[1-{ }^{13} \mathrm{C}\right]$ pyruvate to $\left[1-{ }^{13} \mathrm{C}\right]$ lactate exchange, together with decreased lactate dehydrogenase activity, were found in glioblastoma xenografts and orthotopic tumor models following treatment with the PI3K inhibitor LY294002 or the mTOR inhibitor Everolimus $^{21}$.

\subsubsection{Tricarboxylic Acid Cycle metabolism}

Recently there has been renewed interest in glutamine metabolism and that of various tricarboxylic acid (TCA)-cycle intermediates in cancer. An MRS study of ${ }^{13} \mathrm{C}$ labelled substrates showed that glutamine was used as the major anaplerotic precursor 
in proliferating cancer cells ${ }^{22}$. In tumors with mutations of the TCA-cycle enzymes succinate dehydrogenase and fumarate hydratase $(\mathrm{FH})$ the intermediates succinate and fumarate accumulate. Both of these have been found to act as oncometabolites that enhance tumor growth and survival during stress by mechanisms that include activation of HIF-1. Increases in fumarate level and lactate production, together with upregulated HIF-1 target genes, were observed in FH-deficient tissues isolated from FH-deficient mice ${ }^{23}$, which is consistent with the ${ }^{1} \mathrm{H}$-MRS metabolic profiles of $\mathrm{FH}$ deficient cells ${ }^{24}$.

Mutation of the TCA cycle enzyme isocitrate dehydrogenase (IDH1) induces a gain of function allowing it to synthesize the oncometabolite 2-hydroxyglutarate (2HG) instead of $\alpha$-ketoglutarate $(\alpha-K G) .2-H G$ accumulates at millimolar concentrations in some gliomas and can be detected by ${ }^{1} \mathrm{H}$ MRS in vivo ${ }^{25}$. In vivo ${ }^{13} \mathrm{C}$ MRS of hyperpolarized $\left[1-{ }^{13} \mathrm{C}\right] \alpha-\mathrm{KG}$ has also been used on gliomas to examine the effect of IDH1 gene mutation on the formation of 2-HG. This mutation is associated with a decrease in the activity of branched-chain amino acid transaminase-1 (BCAT1), the enzyme that catalyzes the transamination of branched-chain amino acids while converting $\alpha-\mathrm{KG}$ to glutamate. Only $\left[1-{ }^{13} \mathrm{C}\right] \alpha-\mathrm{KG}$ was observed in IDH1 wild-type glioma tumors, whereas, hyperpolarized $\left[1-{ }^{13} \mathrm{C}\right] 2-\mathrm{HG}$ was found in IDH1 mutant orthotopic tumors ${ }^{26}$. A lower conversion rate of hyperpolarized $\left[1-{ }^{13} \mathrm{C}\right] \alpha-\mathrm{KG}$ to hyperpolarized $\left[1-{ }^{13} \mathrm{C}\right]$ glutamate was also found in the IDH1 mutant tumors, consistent with reduced BCAT1 activity ${ }^{27}$.

\subsubsection{Tumor bioenergetics and $\mathrm{pH}$}


In vivo ${ }^{31} \mathrm{P}$ MRS has been used to study tumor bioenergetics and tumor $\mathrm{pH}$ noninvasively, by measuring metabolites such as nucleoside triphosphates (NTP), phosphocreatine $(\mathrm{PCr})$ and inorganic phosphate $(\mathrm{Pi})$. Intracellular $\mathrm{pH}(\mathrm{pHi})$ is measured from the chemical shift between the $\mathrm{Pi}$ resonance ( $\mathrm{pH}$-sensitive) and a $\mathrm{pH}$ insensitive resonance of a metabolite such as, PCr or NTP (see emrstm1458). A decrease in high-energy phosphates (NTP and PCr) and increased Pi, together with a more acidic pHi have been found in hypoxic tumors. Hypoxia can develop when tumor growth exceeds the blood supply or when reduced micro-vessel density develops following therapy ${ }^{28}$.

\subsubsection{Membrane metabolism}

Increased growth and proliferation in tumors requires elevated membrane synthesis and turnover. Phospholipids are major membrane components and are also involved in the regulation of cell function and signaling pathways. Choline-containing phospholipid membrane components can be detected by MRS, and they have been used to aid diagnosis and prognosis in cancer $^{29}$ (see also emrstm1425). In vivo ${ }^{1} \mathrm{H}$ MRS provides information on the total choline level, which includes signals from free choline, phosphoethanolamine (PE), PC, GPC and glycerophosphoethanolamine (GPE), whereas in vivo ${ }^{31} \mathrm{P}$ MRS detects a peak from phosphomonoesters (PME, comprised of PE and PC) and another peak form phosphodiesters (PDE, comprised of GPC and GPE). An increase in PME has been attributed to increased membrane synthesis, often associated with rapid tissue growth, whereas the PDE resonance is associated with membrane breakdown. Increased choline-containing compounds or the total choline level have been used as endogenous biomarkers that distinguished between benign and malignant tumors in many types of human cancers ${ }^{29}$. Assessment 
of phospholipid metabolism by MRS could also provide insights into tumor response following treatment (emrstm1093).

$\mathrm{PC}$ is involved in cell membrane synthesis and is formed by the phosphorylation of choline, catalyzed by choline kinase (ChoK). Increased levels of PC and ChoK are associated with cell proliferation and malignant transformation in tumors, and inhibition of ChoK results in reduced PC levels ${ }^{29}$. Changes in phospholipid metabolites have also been found by MRS, both in vivo and in tumor extracts, following treatment with conventional cytotoxic therapies and experimental anticancer drugs (emrstm1093). Reduced PC was also found in tumors with HIF-1 deficiency when compared with wild-type controls (emrstm1093).

\subsubsection{Lipid metabolism}

MR-visible lipid (or mobile lipid) signals arise from cytosolic lipid vesicles that are present in both proliferating and malignant cells; they have been found by ${ }^{1} \mathrm{H}$ MRS in a variety of tumors, both in vivo and in tissue extracts ex vivo. The MR-visible lipids are comprised of triglycerides and cholesterol esters in intracellular neutral lipid droplets. Increased lipid signals from droplets are found in tumors under stress or undergoing apoptosis and necrosis following therapies. The underlying reasons for the higher level of lipids and the mechanisms that control the production of lipid droplets are currently under investigation ${ }^{6}$. It has been suggested that they may play a role in detoxifying the cells under stress or act as an alternative energy source ${ }^{6}$.

Inhibition of fatty acid synthase, an enzyme that catalyzes the terminal steps in the synthesis of saturated fatty acids, by the fatty acid synthase inhibitor Orlisat 
resulted in reduced levels of fatty acids as measured by ${ }^{1} \mathrm{H}$ and ${ }^{31} \mathrm{P}$ MRS(emrstm1093).

\subsection{Cardiovascular Disease}

Metabolomic studies by MRS have been used in cardiovascular diseases to develop biomarkers for diagnosis and prognosis, for predicting disease progression and treatment response, and for identifying treatment targets ${ }^{30}$. In vivo ${ }^{1} \mathrm{H}$ MRS can be used to measure the total creatine level in heart tissues and in vivo ${ }^{31} \mathrm{P}$ MRS is used to examine heart bioenergetics and creatine kinase flux in cardiac diseases and dysfunction $^{31,32}$. Intramyocardial triglyceride content in human subjects can also be measured by in vivo ${ }^{1} \mathrm{H}$ MRS [see emrstm1453]. HR-MAS MRS has identified taurine as a potential biomarker for myocardial infarction ${ }^{33}$ and this method has also been used to examine the acute effect of 3,4-methylenedioxymethamphetamine on cardiac metabolism in the $\mathrm{rat}^{34}$. Non-invasive DNP studies are used to assess metabolism in cardiovascular disease in vivo ${ }^{35,36} \cdot\left[1-{ }^{13} \mathrm{C}\right]$ pyruvate has been the most widely studied substrate to date because of its central role in cellular metabolism. Combined used of hyperpolarized $\left[1-{ }^{13} \mathrm{C}\right]-$ and $\left[2-{ }^{13} \mathrm{C}\right]$-pyruvate has resulted in significant advances in the understanding of real time myocardial metabolism in the normal and diseased heart in vivo, as TCA cycle metabolism can also be assessed ${ }^{35,36}$. In addition to pyruvate, hyperpolarized ${ }^{13} \mathrm{C}$-labelled bicarbonate has been used to measure extracellular $\mathrm{pH}$ in the perfused rat heart after ischemia ${ }^{35-36}$.

${ }^{1} \mathrm{H}$ MRS based metabolomics has been combined with proteomics in the investigation of gene manipulation and ischemic preconditioning of smooth muscle cells and heart tissues; it showed that a deficiency of either PKC- $\varepsilon$ or PKC- $\delta$ was 
sufficient to abrogate the cardioprotective effects of ischemic preconditioning ${ }^{37,38}$. In another study from the same group, proteomic and ex vivo ${ }^{1} \mathrm{H}$ MRS metabolomic analysis of aortas from apolipoprotein $\mathrm{E}(\mathrm{apoE})$ mice demonstrated that inefficient vascular glucose and energy metabolism coincides with increased oxidative stress in hyperlipidemic animals, as evidenced by oxidation of redox-sensitive proteins ${ }^{39}$. Similar studies combining ${ }^{1} \mathrm{H}$ MRS-based metabolomics with proteomics have been performed on biopsies from the human heart ${ }^{40}$.

\subsection{Neurological Disorders and Neurodegenerative Diseases}

Localized in vivo ${ }^{1} \mathrm{H}$ MRS can detect many metabolites non-invasively in human brain, including N-acetylaspartate (NAA), creatine and phosphocreatine, choline, myoinositol, lipids, lactate and glutamate/glutamine/GABA ${ }^{41}$ (see also emrstm1449). NAA and myo-inositol are regarded as neuronal and glial biomarkers, respectively, while creatine and phosphocreatine are involved in the bioenergetic pathways and the choline signal provides information about membrane turnover. Glutamate is a neurotransmitter and plays an important role in brain function ${ }^{42}$ while lactate is involved in the glycolytic activity of the brain. These MRS-detectable metabolites are found to alter in many neurological disorders and neurodegenerative diseases, both in human patients and in animal models, e.g., brain injuries, stroke, Parkinson's disease, multiple sclerosis, schizophrenia, Alzheimer's disease and) prion diseases such as transmissible spongiform encephalopathy ${ }^{43-46}$. In vivo ${ }^{31} \mathrm{P}$ MRS has been used to assess the bioenergetic status of the brain, as the high-energy phosphates (PCr and NTP), Pi and intracellular $\mathrm{pH}$ (as noted above) are modulated in neurological conditions such as hypoxic-ischemic brain injury ${ }^{43}$. 
In tissues of individuals with schizophrenia, ${ }^{1} \mathrm{H}$ HR-MAS MRS combined with proteomic and transcriptomic data have shown increased glycolytic flux when compared to the corresponding tissues from individuals with bipolar disorder ${ }^{47}$. In a study of ex vivo ${ }^{1} \mathrm{H}$ HR-MAS MRS of human brain biopsies, Cheng et al showed a linear relationship between neuronal count (neuronal density) and NAA, and found that metabolite ratios are better than neuronal counts in the diagnosis of Alzheimer's disease $^{48}$. Further details concerning the use of MRS to monitor metabolism in neurology and neurological diseases can be found in emrstm1449, emrstm1464, emrstm1491 and emrstm1463.

\subsection{Muscle Diseases}

In vivo ${ }^{31} \mathrm{P}$ MRS is commonly used to assess the bioenergetic status and function of resting and exercising muscle, as resting muscle contains high levels of $\mathrm{PCr}$ and ATP, and during exercise the muscle $\mathrm{PCr}$ level and intracellular $\mathrm{pH}$ decrease, accompanied by an increase in muscle $\mathrm{Pi}^{49}$. Studies have been performed during rest and exercise in many diseases, e.g., muscular dystrophy; myositis and scleroderma ${ }^{50-53}$, and to monitor treatment response $\mathrm{e}^{54}$. The absolute concentrations of phosphorylated metabolites in muscles with different fiber composition have been determined in vivo by localized ${ }^{31} \mathrm{P}$ MRS in the rat hind limb ${ }^{55}$. Attempts have also been made to quantify the absolute concentration of phosphorus metabolites in human muscle ${ }^{56} \cdot{ }^{1} \mathrm{H}$ MRS has been applied to study lipid metabolism in muscle. ${ }^{1} \mathrm{H}$ MRS can measure intramyocellular lipids ${ }^{57}$ which have been found to play a role in the development of insulin resistance ${ }^{58}$ and metabolic abnormalities ${ }^{59}$. Further details concerning the use of MRS for examining muscle metabolism can be found in emrstm1442 and emrstm1431. 



\section{BIOSKETCHES}

John Griffiths MB BS, D Phil, FRCP, qualified in medicine and biochemistry. In the early 1980s, his research group pioneered the use of MRS for studies on living tumors, and he has worked since then on MRI and MRS of cancer, both in vivo and ex vivo. He has published more than 300 peer-reviewed articles to date. His recent interests include the metabolomics of cancer.

Madhu Basetti. MSc 1983, PhD (physics) 1989, Osmania University, Hyderabad, India. Worked in Germany and Sweden with MRS and MRI in biomedical research before joining John Griffiths' group in London UK in 2000 and (from 2006) Cambridge. Research interests: Application of MRS and MRI methods and NMR based metabolomics for understanding the metabolism and biology of cancer. He has published about 52 peer- reviewed articles to date.

Yuen-Li Chung BSc, PhD in Chemistry. She has applied MRI and MRS to study neurodegenerative, muscular and cardiovascular diseases. Since the year 2000, she is specialised in using a range of MRS techniques (including metabolomics) to investigate cellular processes in the metabolism of cancer and its response to treatment both in vivo and in vitro. She has published about 65 peer-reviewed articles to date. 


\begin{tabular}{|c|c|c|c|}
\hline \multicolumn{4}{|c|}{ Metabolites Quantifiable by ${ }^{1} \mathrm{H}$ MRS ex vivo } \\
\hline \multicolumn{4}{|c|}{ Glycolytic Metabolites } \\
\hline$\alpha$-Glucose & $\beta$-Glucose & Lactate & Pyruvate \\
\hline \multicolumn{4}{|c|}{ Amino Acids and Associated Metabolites } \\
\hline Alanine & Asparagine & Aspartic Acid & Glutamate \\
\hline Glutamine & Glycine & Histidine & iso-Leucine \\
\hline Leucine & Lysine & Phenylalanine & Serine \\
\hline Threonine & Tyrosine & Taurine & Valine \\
\hline$\alpha$-Ketoisovalerate & Glutathione & $\mathrm{N}$-acetyl aspartate & \\
\hline \multicolumn{4}{|c|}{ Membrane-Associated Metabolites } \\
\hline Choline & Phosphocholine & Glycerophosphocholine & Phosphoethanolamine \\
\hline Dimethylamine & Betaine & Cholesterols \& esters & Phosphatidylcholine \\
\hline $\begin{array}{l}\text { Phosphatidyl- } \\
\text { ethanolamine }\end{array}$ & $\begin{array}{l}\text { Phosphatidyl- } \\
\text { glycerol }\end{array}$ & Glycerol & \\
\hline Plasmalogen & Triacylglycerol & Myo-inositol & Scyllo-inositol \\
\hline Energy Metabol & & & \\
\hline
\end{tabular}




\begin{tabular}{|l|l|l|l|}
\hline ATP \& ADP & Creatine & Phosphocreatine & NAD $^{+}$\& NADH \\
\hline Inosine & UTP \& UDP & & \\
\hline \multicolumn{3}{|l|}{} \\
TCA Cycle \& Related Metabolites & Succinate & Acetate \\
\hline$\beta$-hydroxybutyrate & Fumarate & & \\
\hline Citrate & & & \\
\end{tabular}

Table 1. Metabolites routinely quantifiable by ${ }^{1} \mathrm{H}$ MRS in living tissues. 
Table 2. Databases used in metabolomics studies

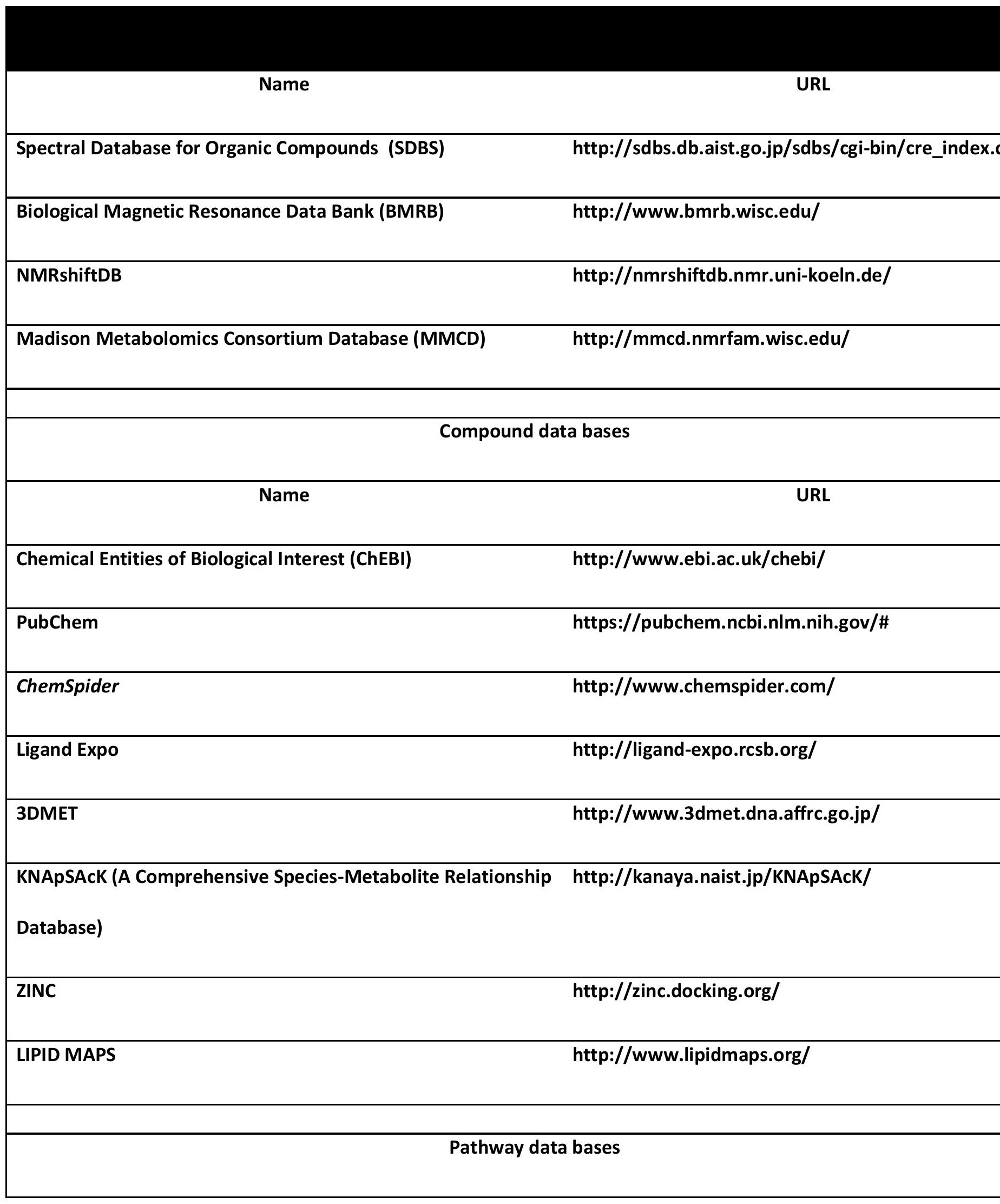




\begin{tabular}{|c|c|}
\hline Name & URL \\
\hline KEGG: Kyoto Encyclopedia of Genes and Genomes & http://www.genome.jp/kegg/ \\
\hline The Small Molecule Pathway Database (SMPDB ) & http://smpdb.ca/ \\
\hline BioCyc & http://biocyc.org/ \\
\hline MetaCyc Metabolic Pathway Database & http://metacyc.org/ \\
\hline Reactome & http://www.reactome.org/ \\
\hline \multicolumn{2}{|c|}{ Comprehensive metabolomic data bases } \\
\hline Name & URL \\
\hline KEGG: Kyoto Encyclopedia of Genes and Genomes & http://www.genome.jp/kegg/ \\
\hline The Human Metabolome Database (HMDB) & http://www.hmdb.ca/ \\
\hline Madison Metabolomics Consortium Database (MMCD) & http://mmcd.nmrfam.wisc.edu/ \\
\hline DrugBank database & http://www.drugbank.ca/ \\
\hline
\end{tabular}




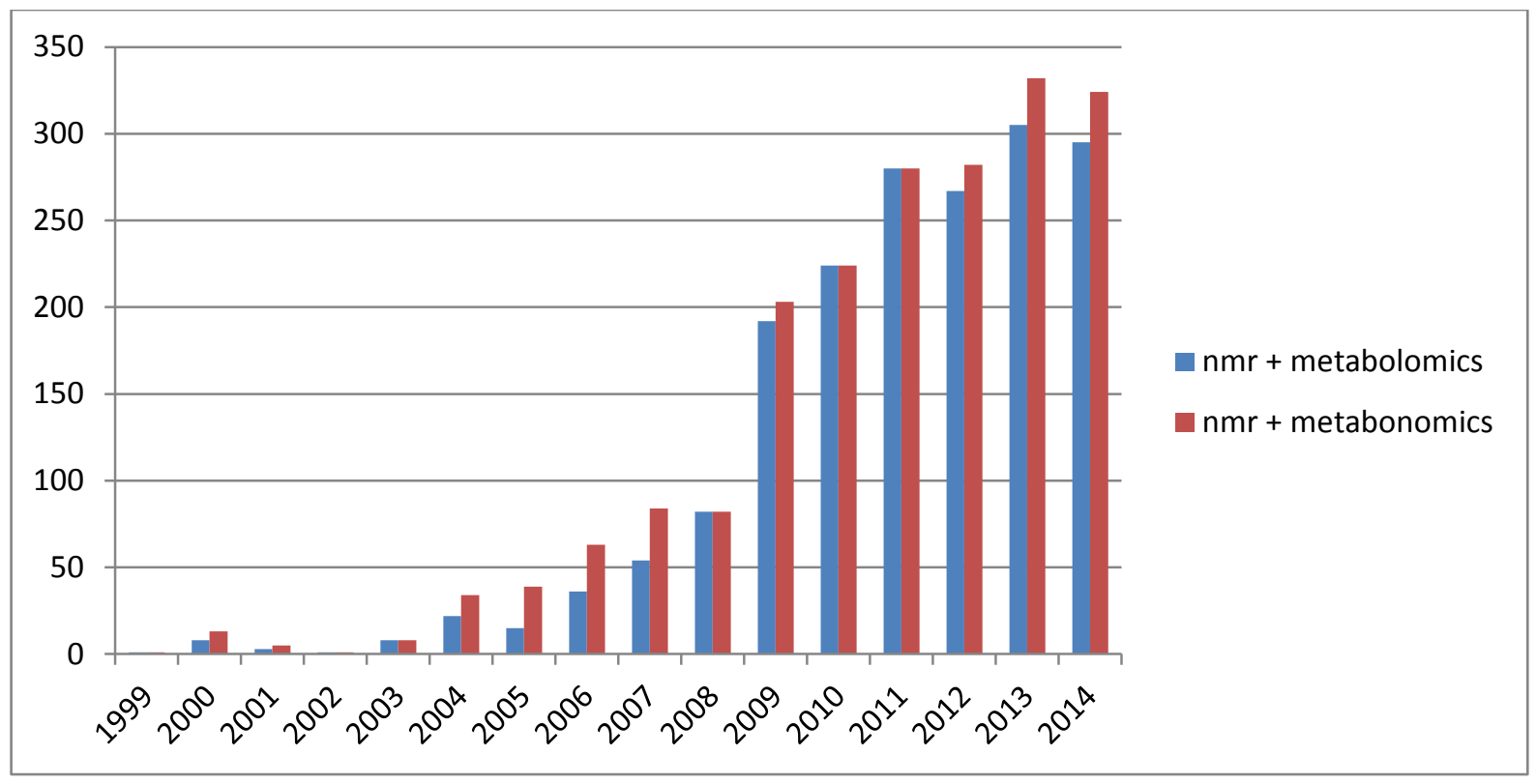

Figure 1. PubMed data showing articles with the search words NMR and metabolomics or NMR and metabonomics. 

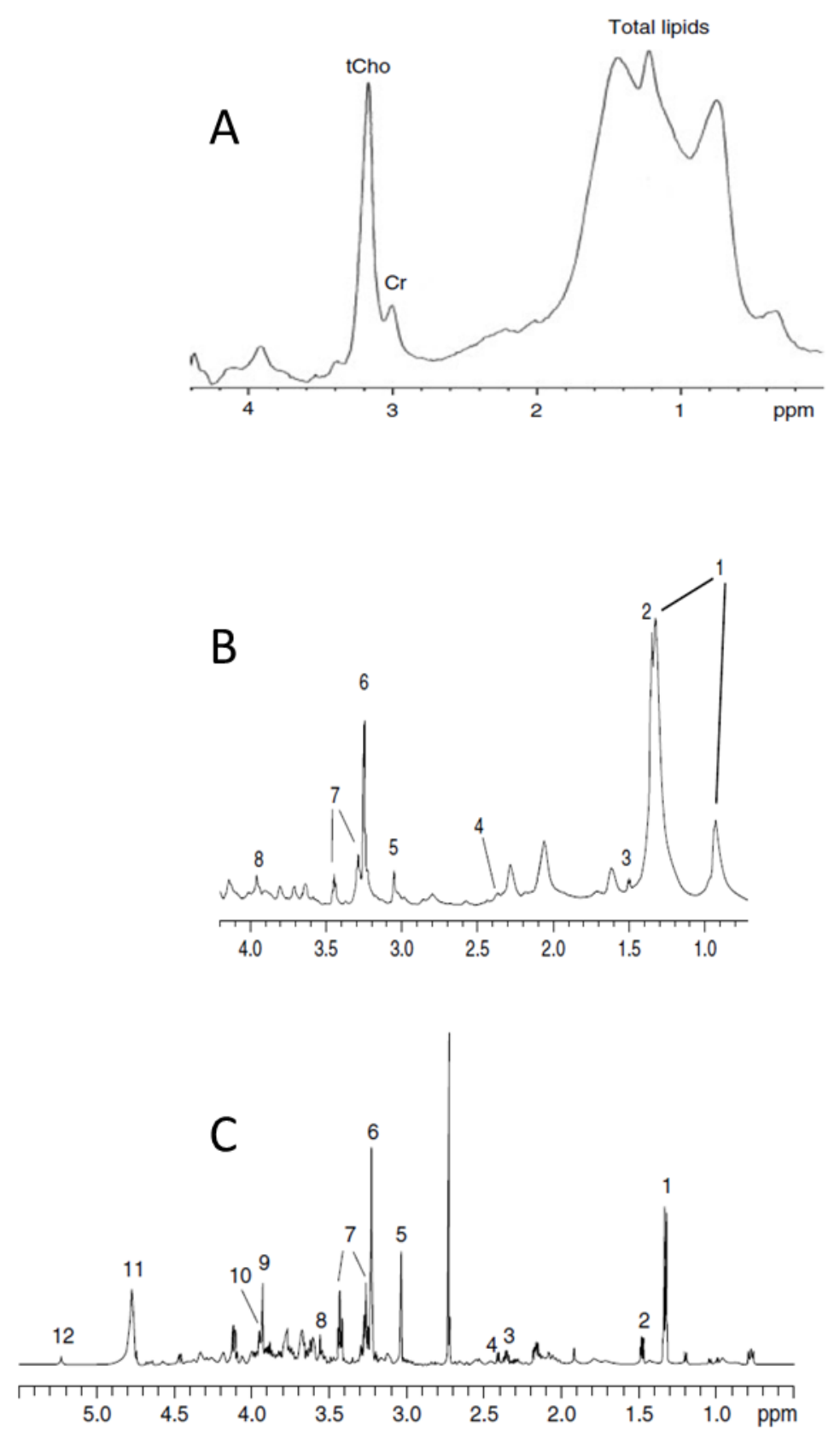

Figure 2. ${ }^{1} \mathrm{H}$ spectra of HT29 tumors, illustrating the improved resolution available from ex vivo spectra obtained by HR-MAS and tissue extraction. A. In vivo localized ${ }^{1} \mathrm{H}$ MR spectrum of an HT29 tumor in a mouse. tCho, total choline; Cr, creatine. B. HR-MAS ${ }^{1} \mathrm{H}$ spectrum of an excised HT29 tumor. 1, lipids; 2, lactate; 3, 
alanine; 4 , glutamate; 5 , creatine + phosphocreatine; 6 , choline-containing compounds; 7 , taurine; 8 , creatine + phosphocreatine. C. In vitro ${ }^{1} \mathrm{H}$ MR spectrum of a perchloric acid extract of a HT29 tumor. 1, lactate; 2, alanine; 3, glutamate; 4, Glutamine; 5 , creatine + phosphocreatine; 6 , phosphocholine+ glycerophosphocholine; 7, taurine; 8, glycine; 9, creatine; 10, phosphocreatine; 11 , water; 12, glucose. Adapted from YL Chung \& JR Griffiths, Metabolomic Studies on Cancer and on Anticancer Drugs by NMR Ex Vivo, emrstm1093. 


\section{PCA}
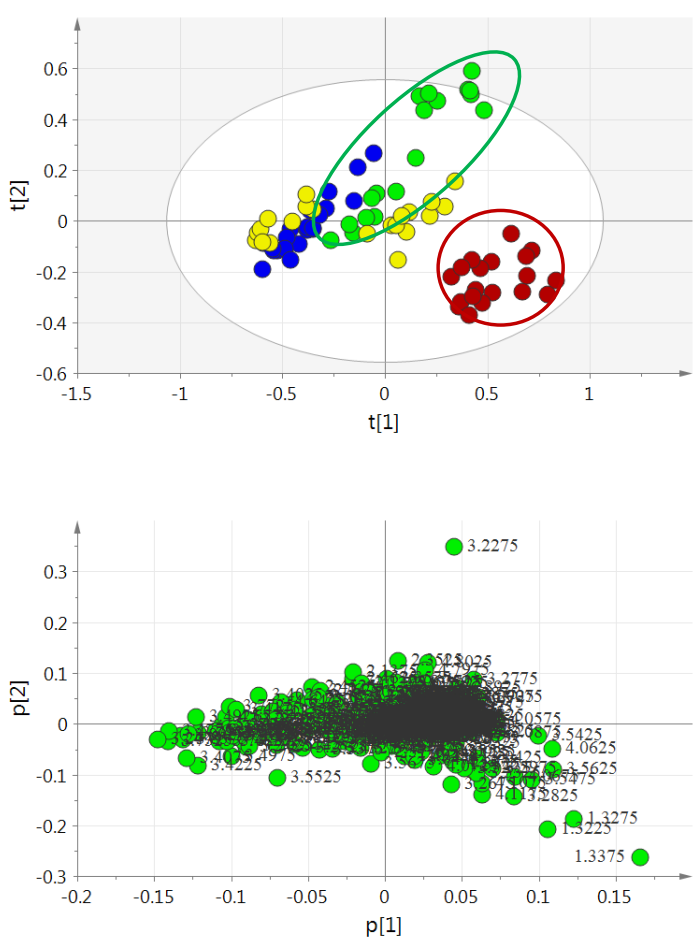

OPLS-DA
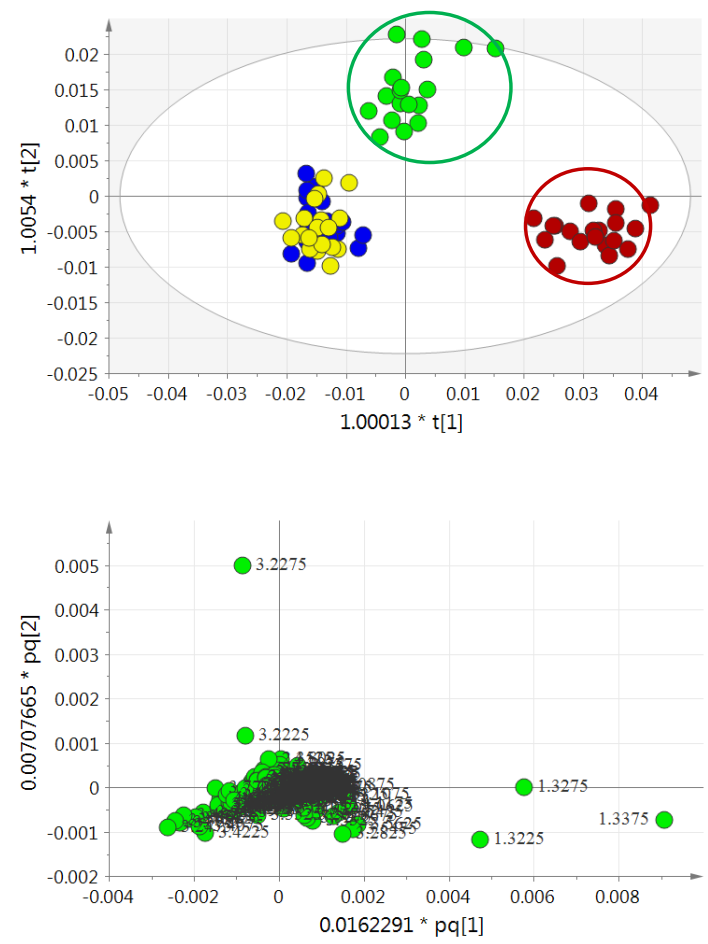

Figure 3. PCA and OPLS-DA analysis of ${ }^{1} \mathrm{H}$ NMR metabolite extract data from wild type and HIF-1a knockout astrocyte cells grown in normoxic and hypoxic conditions. Top panels: PCA and OPLS-DA scores plots from wild type (yellow-normoxia and red hypoxia) and HIF-1a knockout astrocytes (Blue - normoxia and Green - hypoxia). There is a clearer separation of wild type and knock-out astrocyte cells grown at hypoxic conditions in the OPLS-DA plot (red and green circles). Bottom panels: loadings plots from both methods show that lactate and phosphocholine are mainly responsible for the separation of clusters in hypoxia. 


\section{References:}

1. B. Madhu, M. Dadulescu and J. Griffiths, MAGMA, 2014, 28, 161.

2. J. Folch, M. Lees and G. H. Sloane Stanley, J Biol Chem, 1957, 226, 497.

3. J. Henke, W. Willker, J. Engelmann and D. Leibfritz, Anticancer Res, 1996, 16, 1417.

4. O. Beckonert, M. Coen, H. C. Keun, Y. Wang, T. M. Ebbels, E. Holmes, J. C. Lindon and J. K. Nicholson, Nat Protoc, 2010, 5, 1019.

5. B. Worley and R. Powers, Current Metabolomics, 2013, 1, 92.

6. E. J. Delikatny, S. Chawla, D. J. Leung and H. Poptani, NMR Biomed, 2011, $24,592$.

7. S. E. Day, M. I. Kettunen, F. A. Gallagher, D. E. Hu, M. Lerche, J. Wolber, K. Golman, J. H. Ardenkjaer-Larsen and K. M. Brindle, Nat Med, 2007, 13, 1382.

8. G. Lin, D. K. Hill, G. Andrejeva, J. K. Boult, H. Troy, A. C. Fong, M. R. Orton, R. Panek, H. G. Parkes, M. Jafar, D. M. Koh, S. P. Robinson, I. R. Judson, J. R. Griffiths, M. O. Leach, T. R. Eykyn and Y. L. Chung, Br J Cancer, 2014, 111, 375.

9. G. Lin, G. Andrejeva, A. C. Wong Te Fong, D. K. Hill, M. R. Orton, H. G. Parkes, D. M. Koh, S. P. Robinson, M. O. Leach, T. R. Eykyn and Y. L. Chung, PLoS One, 2014, 9, e92645.

10. D. Hanahan and R. A. Weinberg, Cell, 2011, 144, 646.

11. A. Schulze and A. L. Harris, Nature, 2012, 491, 364.

12. M. S. Palmnas and H. J. Vogel, Metabolites, 2013, 3, 373.

13. B. K. Sarkar, C. Chakraborty, A. R. Sharma, K. J. Bae, G. Sharma, G. P. Doss, D. Dutta, S. Ding, B. Ganbold, J. S. Nam and S. S. Lee, Front Biosci (Landmark Ed), 2014, 19, 1186.

14. M. Bulik, R. Jancalek, J. Vanicek, A. Skoch and M. Mechl, Clin Neurol Neurosurg, 2013, 115, 146. 15. S. Kundu, S. Chopra, A. Verma, U. Mahantshetty, R. Engineer and S. K. Shrivastava, J Cancer Res Ther, 2012, 8, 11.

16. W. Chen, Y. Zu, Q. Huang, F. Chen, G. Wang, W. Lan, C. Bai, S. Lu, Y. Yue and F. Deng, Magn Reson Med, 2011, 66, 1531.

17. P. J. Bolan, Magn Reson Imaging Clin N Am, 2013, 21, 625.

18. M. F. Penet, M. M. Gadiya, B. Krishnamachary, S. Nimmagadda, M. G. Pomper, D. Artemov and Z. M. Bhujwalla, Cancer Res, 2011, 71, 6948.

19. M. Stubbs, P. M. McSheehy, J. R. Griffiths and C. L. Bashford, Mol Med Today, 2000, 6, 15.

20. S. Biswas, H. Troy, R. Leek, Y. L. Chung, J. L. Li, R. R. Raval, H. Turley, K. Gatter, F. Pezzella, J. R. Griffiths, M. Stubbs and A. L. Harris, J Oncol, 2010, 2010, 757908.

21. C. S. Ward, H. S. Venkatesh, M. M. Chaumeil, A. H. Brandes, M. Vancriekinge, H. Dafni, S.

Sukumar, S. J. Nelson, D. B. Vigneron, J. Kurhanewicz, C. D. James, D. A. Haas-Kogan and S. M. Ronen, Cancer Res, 2010, 70, 1296.

22. R. J. DeBerardinis, A. Mancuso, E. Daikhin, I. Nissim, M. Yudkoff, S. Wehrli and C. B. Thompson, Proc Natl Acad Sci U S A, 2007, 104, 19345.

23. H. Ashrafian, L. O'Flaherty, J. Adam, V. Steeples, Y. L. Chung, P. East, S. Vanharanta, H. Lehtonen, E. Nye, E. Hatipoglu, M. Miranda, K. Howarth, D. Shukla, H. Troy, J. Griffiths, B. Spencer-Dene, M. Yusuf, E. Volpi, P. H. Maxwell, G. Stamp, R. Poulsom, C. W. Pugh, B. Costa, C. Bardella, M. F. Di Renzo, M. I. Kotlikoff, V. Launonen, L. Aaltonen, M. El-Bahrawy, I. Tomlinson and P. J. Pollard, Cancer Res, 2010, 70, 9153.

24. W. M. Linehan and T. A. Rouault, Clin Cancer Res, 2013, 19, 3345.

25. C. Choi, S. K. Ganji, R. J. DeBerardinis, K. J. Hatanpaa, D. Rakheja, Z. Kovacs, X. L. Yang, T. Mashimo, J. M. Raisanen, I. Marin-Valencia, J. M. Pascual, C. J. Madden, B. E. Mickey, C. R. Malloy, R. M. Bachoo and E. A. Maher, Nat Med, 2012, 18, 624.

26. M. M. Chaumeil, P. E. Larson, H. A. Yoshihara, O. M. Danforth, D. B. Vigneron, S. J. Nelson, R. O. Pieper, J. J. Phillips and S. M. Ronen, Nat Commun, 2013, 4, 2429.

27. M. M. Chaumeil, P. E. Larson, S. M. Woods, L. Cai, P. Eriksson, A. E. Robinson, J. M. Lupo, D. B. Vigneron, S. J. Nelson, R. O. Pieper, J. J. Phillips and S. M. Ronen, Cancer Res, 2014, 74, 4247. 
28. Y. L. Chung, H. Troy, R. Kristeleit, W. Aherne, L. E. Jackson, P. Atadja, J. R. Griffiths, I. R. Judson, P. Workman, M. O. Leach and M. Beloueche-Babari, Neoplasia, 2008, 10, 303.

29. K. Glunde, Z. M. Bhujwalla and S. M. Ronen, Nat Rev Cancer, 2011, 11, 835.

30. J. L. Griffin, H. Atherton, J. Shockcor and L. Atzori, Nat Rev Cardiol, 2011, 8, 630.

31. C. J. Holloway, J. Suttie, S. Dass and S. Neubauer, Prog Cardiovasc Dis, 2011, 54, 320.

32. R. Beadle and M. Frenneaux, Expert Rev Cardiovasc Ther, 2010, 8, 269.

33. Y. Yang, L. Yang, Y. Zhang, X. Gu, D. Xu, F. Fang, A. Sun, K. Wang, Y. Yu, J. Zuo and J. Ge, Exp Ther Med, 2013, 5, 683.

34. S. A. Perrine, M. S. Michaels, F. Ghoddoussi, E. M. Hyde, M. E. Tancer and M. P. Galloway, NMR Biomed, 2009, 22, 419.

35. O. J. Rider and D. J. Tyler, J Cardiovasc Magn Reson, 2013, 15, 93.

36. M. A. Schroeder, K. Clarke, S. Neubauer and D. J. Tyler, Circulation, 2011, 124, 1580.

37. M. Mayr, B. Metzler, Y. L. Chung, E. McGregor, U. Mayr, H. Troy, Y. Hu, M. Leitges, O. Pachinger, J. R. Griffiths, M. J. Dunn and Q. Xu, Am J Physiol Heart Circ Physiol, 2004, 287, H946.

38. M. Mayr, Y. L. Chung, U. Mayr, E. McGregor, H. Troy, G. Baier, M. Leitges, M. J. Dunn, J. R.

Griffiths and Q. Xu, Am J Physiol Heart Circ Physiol, 2004, 287, H937.

39. Mayr M, Zampetaki A, Sidibe A, Mayr U, Yin X, De Souza Al, Chung YL, Madhu B, Quax PH, Hu Y, Griffiths JR, Xu Q. Circ Res. 2008, 102, 1046.

40. M. Mayr, S. Yusuf, G. Weir, Y. L. Chung, U. Mayr, X. Yin, C. Ladroue, B. Madhu, N. Roberts, A. De Souza, S. Fredericks, M. Stubbs, J. R. Griffiths, M. Jahangiri, Q. Xu and A. J. Camm, J Am Coll Cardiol, 2008, 51, 585.

41. M. van der Graaf, Eur Biophys J, 2010, 39, 527.

42. S. Ramadan, A. Lin and P. Stanwell, NMR Biomed, 2013, 26, 1630.

43. L. S. de Vries and F. Groenendaal, Neuroradiology, 2010, 52, 555.

44. S. L. Risacher and A. J. Saykin, Semin Neurol, 2013, 33, 386.

45. B. R. Sajja, J. S. Wolinsky and P. A. Narayana, Neuroimaging Clin N Am, 2009, $19,45$.

46. N. Zhang, X. Song, R. Bartha, S. Beyea, R. D'Arcy, Y. Zhang and K. Rockwood, Curr Alzheimer Res, 2014, 11, 367.

47. S. Prabakaran, J. E. Swatton, M. M. Ryan, S. J. Huffaker, J. T. Huang, J. L. Griffin, M. Wayland, T. Freeman, F. Dudbridge, K. S. Lilley, N. A. Karp, S. Hester, D. Tkachev, M. L. Mimmack, R. H. Yolken, M. J. Webster, E. F. Torrey and S. Bahn, Mol Psychiatry, 2004, 9, 684.

48. L. L. Cheng, K. Newell, A. E. Mallory, B. T. Hyman and R. G. Gonzalez, Magn Reson Imaging, 2002, 20, 527.

49. A. M. Jones, D. P. Wilkerson, F. DiMenna, J. Fulford and D. C. Poole, Am J Physiol Regul Integr Comp Physiol, 2008, 294, R585.

50. J. A. Kent-Braun, R. G. Miller and M. W. Weiner, Radiol Clin North Am, 1994, 32, 313.

51. Z. Argov and D. L. Arnold, Neurol Clin, 2000, 18, 35, N. J. Olsen, J. H. Park and L. E. King, Jr., Curr Rheumatol Rep, 2001, 3, 346.

52. J. P. Mattei, D. Bendahan and P. Cozzone, Reumatismo, 2004, 56, 9.

53. N. J. Olsen, L. E. King, Jr. and J. H. Park, Rheum Dis Clin North Am, 1996, 22, 783.

54. Y. L. Chung, H. Alexanderson, N. Pipitone, C. Morrison, M. Dastmalchi, C. Stahl-Hallengren, S. Richards, E. L. Thomas, G. Hamilton, J. D. Bell, I. E. Lundberg and D. L. Scott, Arthritis Rheum, 2007, $57,694$.

55. B. Madhu, K. Lagerwall and B. Soussi, NMR Biomed, 1996, 9, 327.

56. G. J. Kemp, M. Meyerspeer and E. Moser, NMR Biomed, 2007, 20, 555.

57. C. Boesch and R. Kreis, Ann N Y Acad Sci, 2000, 904, 25.

58. J. Machann, N. Stefan and F. Schick, Eur J Radiol, 2008, 67, 275.

59. J. Dube and B. H. Goodpaster, Curr Opin Clin Nutr Metab Care, 2006, 9, 553. 